\title{
Distribuição e alimentação de duas espécies simpátricas de piranhas Serrasalmus maculatus e Pygocentrus nattereri (Characidae, Serrasalminae) do rio Ibicuí, Rio Grande do Sul, Brasil
}

\author{
Everton R. Behr ${ }^{1} \&$ Cleiton A. Signor ${ }^{2}$
}

1. Centro de Educação Superior Norte do Rio Grande do Sul, Universidade Federal de Santa Maria. Av, Independência, 3751, 98300-000 Palmeira das Missões, RS. (everton_behr@hotmail.com)

2. Programa de Pós-Graduação em Ecologia e Conservação da Biodiversidade, Universidade Federal do Mato Grosso, Instituto de Biociências. Av. Fernando Corrêa da Costa, s/n, CCBS-II, Boa Esperança, 78060-900 Cuiabá, MT. (cleitonsignor@yahoo.com.br)

\begin{abstract}
Distribution and feeding of two sympatric species of piranhas Serrasalmus maculatus and Pygocentrus nattereri (Characidae, Serrasalminae) of the Ibicuí river, State of Rio Grande do Sul, Brazil. Two species of piranhas occur in the Ibicuí River, one of the main tributaries of the Uruguay River in southern Brazil, Serrasalmus maculatus Kner, 1858 and Pygocentrus nattereri Kner, 1858. In order to analyze the distribution and feeding habits of these species we conducted collecting expeditions from December 1999 to January 2002, using a variety of fishing gears in three sites along the river, both in lentic and lotic habitats. A total of 203 individuals of $S$. maculatus and 86 of $P$. nattereri were caught, mostly in lentic habitats and closer to the Uruguay River, were $P$. nattereri seems to be more concentrated. The number of individuals captured at different times did not show variation in $P$. nattereri while the number of $S$. maculatus captured at mid-night was the highest. Young $S$. maculatus (2-4 cm standard length) fed mainly on fish fins and insects. Fish was the predominant item in remaining size classes for both species, with intra- and inter-specific overlap in larger classes $(8-16 \mathrm{~cm}$ and $>16 \mathrm{~cm}$ standard length). No differences in the repletion index between day time or habitats, were observed for the two species.
\end{abstract}

KEYWORDS. Serrasalmus maculatus, Pygocentrus nattereri, distribution, feeding, Ibicuí River.

RESUMO. Duas espécies de piranhas ocorrem no rio Ibicuí, um dos principais afluentes do rio Uruguai. Com o objetivo de analisar a distribuição e a alimentação destas espécies, foram realizadas coletas de dezembro de 1999 a janeiro de 2002, utilizando-se diferentes artes de pesca em três pontos do rio, sendo que cada ponto foi amostrado em dois ambientes (lêntico e lótico). Foram capturados 203 indivíduos de Serrasalmus maculatus Kner, 1858 e 86 de Pygocentrus nattereri Kner, 1858, sendo que a maior captura de ambas as espécies ocorreu no ambiente lêntico e próximo ao rio Uruguai, onde P. nattereri parece estar mais concentrada. O número de exemplares capturados por horário não apresentou variações significativas em $P$. nattereri enquanto que $S$. maculatus apresentou a maior captura na revisão da meia-noite. Juvenis de $S$. maculatus $(2-4 \mathrm{~cm}$ de comprimento padrão) consumiram preferencialmente nadadeiras e insetos. Nas demais classes de tamanho de ambas as espécies, restos de peixes foi o alimento predominante, havendo sobreposição alimentar intra e interespecífica nas maiores classes $(8-16 \mathrm{~cm}$ e $>16 \mathrm{~cm}$ de comprimento padrão). Não foram constatadas diferenças no índice de repleção entre os horários e os ambientes para as duas espécies.

PALAVRAS-CHAVE. Serrasalmus maculatus, Pygocentrus nattereri, distribuição, alimentação, rio Ibicuí.

No rio Uruguai ocorrem três espécies de piranhas (SverLiJ et al., 1998). Uma destas espécies foi sempre referida na literatura como Serrasalmus spilopleura Kner, 1858. Entretanto, após a revisão realizada por JÉGU (2003), o nome aceito para as bacias dos rios Amazonas e Paraguai-Paraná é Serrasalmus maculatus Kner, 1858. Embora três espécies sejam citadas para o rio Uruguai, no trecho brasileiro são registradas apenas $S$. maculatus e Pygocentrus nattereri Kner, 1858 (Bertoletti, 1985). Na região do alto rio Uruguai, BERTOLETTI et al. (1989, 1990) capturaram apenas $S$. maculatus na área onde foi construída a usina hidrelétrica de Itá e na área da futura usina hidrelétrica de Garabi. Recentemente, a presença de $P$. nattereri foi constatada na região do alto rio Uruguai, embora em número muito menor que $S$. maculatus (E. Zaniboni-Filho, com. pess.).

Serrasalmus maculatus é citada por WeIs et al. (1983) e BOSSEMEYER et al. (1985) para os rios Ibicuí-Mirim e Santa Maria, respectivamente, os quais são afluentes do rio Ibicuí. Os únicos estudos realizados no Rio Grande do Sul exclusivamente com S. maculatus foram aqueles de Beheregaray et al. $(2000,2001)$ na barragem de Sanchuri em Uruguaiana, abordando vários aspectos da biologia da espécie.

As piranhas ou palometas são peixes neotropicais capazes de arrancar pedaços de suas presas e podem atacar animais consideravelmente maiores (MYERS, 1972; Agostinho et al., 1997). Hábitos mutilantes e oportunistas têm sido reportados às piranhas devido ao ataque a diversas espécies de peixes em cativeiro ou no ambiente natural (SAZIMA \& ZAMPROGNO, 1985; NorthCote et al., 1987; Sazima \& Pombal JR., 1988; Sazima \& Machado, 1990; Bistoni \& Haro, 1995; Pompeu, 1999; Oliveira et al., 2004) e em redes de pesca (Agostinho \& Marques, 2001).

Uma dieta onívora com tendência à piscivoria tem sido diagnosticada para várias espécies de piranhas (LEÃo et al., 1991; Almeida et al., 1998; Pompeu, 1999; Agostinho et al., 2003). Os juvenis preferem nadadeiras de peixes, microcrustáceos, insetos e, em algumas espécies, sementes (S AZIma \& Zamprogno, 1985; Machado-Allison \& Garcia, 1986; Nico \& TAPHORN, 
1988; Winemiller, 1989; Nico, 1990; Pompeu, 1999; Alvim et al., 1999). Os adultos ingerem principalmente pedaços de peixes, pequenos peixes inteiros e escamas (NICO \& TAPHORN, 1988; MAGAlHÃes et al., 1990; Bistoni \& HARO, 1995; Almeida et al., 1998; Pompeu, 1999; Agostinho et al., 2003; OliveIRa et al., 2004).

Apesar de várias espécies serem simpátricas em diversos sistemas hidrográficos sul-americanos, poucos trabalhos realizaram comparações entre a dieta destas. Neste contexto, este estudo tem por finalidade analisar a distribuição, a ocupação de habitat, a abundância e a atividade alimentar de $S$. maculatus e $P$. nattereri no rio Ibicuí, bem como comparar a dieta analisando as variações ontogenéticas da dieta das espécies e comparar os padrões de atividade alimentar entre ambientes lóticos e lênticos.

\section{MATERIAL E MÉTODOS}

As coletas foram realizadas em três pontos do rio Ibicuí, afluente do rio Uruguai, no trecho entre os municípios de São Vicente do Sul e Itaqui, correspondendo aos mesmos pontos de amostragem descritos em FAGUNDES et al. (2008). O ponto 1 situa-se pouco abaixo da foz do rio Santa Maria, entre os municípios de São

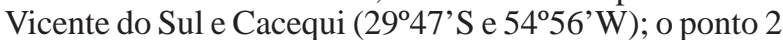
no trecho médio do rio, entre Manoel Viana e Alegrete $\left(29^{\circ} 33^{\prime} \mathrm{S}\right.$ e $\left.55^{\circ} 37^{\prime} \mathrm{W}\right)$ e o ponto 3 , acima da foz do rio Ibirocai, entre Itaqui e Alegrete (292 $5^{\prime}$ S e $\left.56^{\circ} 37^{\prime} \mathrm{W}\right)$. Em cada ponto foram amostrados ambientes lóticos e lênticos constituídos, respectivamente, pela calha principal do rio e por uma lagoa ou canal adjacente. Os ambientes lênticos apresentam, além da distância geográfica entre si, outras características que os diferenciam. No ponto 1 , este ambiente é o canal de um pequeno córrego, mas forma uma lagoa quando a água está alta, localizada a cerca de $40 \mathrm{~m}$ de distância do rio. Em períodos de águas baixas, permanece somente o canal, sem correnteza, com largura variando entre três e oito metros. No ponto 2 a lagoa está conectada ao rio por um estreito e curto canal (dois a três metros de largura e cerca de oito metros de comprimento). Esta é profunda e suas margens são totalmente cobertas por vegetação arbórea e arbustiva, com muitos galhos de árvores caídos em suas margens. Esta lagoa tem cerca de $150 \mathrm{~m}$ de comprimento e $70 \mathrm{~m}$ de largura, ficando isolada do rio em períodos de águas baixas. A lagoa do ponto 3 possui grandes proporções, com praticamente a mesma largura que o rio (em torno de $200 \mathrm{~m}$ ), tendo sua maior largura no ponto em que se liga a ele. Nenhum desses ambientes lênticos apresenta macrófitas aquáticas em abundância.

Foram realizadas coletas bimestrais, de dezembro de 1999 a janeiro de 2002. Para cada ambiente foram utilizados $10 \mathrm{~m}$ de redes de espera com malhas de 1,5;2,0; 2,5 e 3,0 cm; $20 \mathrm{~m}$ de redes de espera com malhas de 4,0; 5,$0 ; 6,0 ; 8,0$ e $10,0 \mathrm{~cm}$; feiticeiras 4,0/20,0; 5,0/20,0 e 6,0/ 20,0 (todas as malhas medidas entre nós adjacentes). Também foram utilizadas redes de arrasto e linhas de mão, entretanto os peixes capturados por estes métodos não foram considerados na análise de captura por ponto, ambiente e horário.

As redes de espera permaneceram na água por $24 \mathrm{~h}$, sendo revisadas a cada seis horas, sempre nos mesmos horários $(6,12,18$ e 24h). As redes de arrasto foram utilizadas duas vezes entre as 16 e $18 \mathrm{~h}$, em locais diferentes. A linha de mão foi utilizada esporadicamente em algumas amostragens. Os peixes coletados foram numerados, fixados com formol a $10 \%$ e depois conservados em álcool $70 \%$ conforme MALABARBA \& REIS (1987). Cada amostra teve seus dados de data, horário de captura, local e aparelho de pesca anotado.

Em laboratório, os peixes foram pesados e medidos (comprimento padrão e comprimento total). O estômago foi retirado e pesado, sendo verificado o grau de repleção e atribuídos pontos conforme a seguinte escala: 0 completamente vazio; 1 - até $25 \%$ do estômago com conteúdo; 2 - acima de $25 \%$ até $75 \%$ do estômago com conteúdo; 3 - acima de $75 \%$ do estômago com conteúdo.

Os estômagos foram abertos para verificação da dieta, sendo utilizados os métodos de freqüência de ocorrência e gravimétrico (HySLOP, 1980; ZAVALA-CAMIN, 1996). Ambos os métodos foram combinados no Índice Alimentar (IAi) (KAWAKAMI \& VAZZOLER, 1980), expresso pela equação:

Onde:

$$
I A i=\frac{F i \cdot P i}{\sum_{i=1}^{n}(F i \cdot P i)} \cdot 100
$$

$I A i=$ Índice Alimentar;

$F i=$ Freqüência relativa do alimento categoria $i$ e

$P i=$ Participação gravimétrica relativa do alimento categoria $i$.

Os conteúdos estomacais foram identificados a olho nu ou com auxílio de lupa. Para identificação dos itens alimentares foram utilizadas chaves dicotômicas, comparação com material de referência e consultas a especialistas. Os itens alimentares foram agrupados em sete categorias: restos de peixes (carne, ossos, escamas); nadadeiras (quando apenas raios estavam presentes, sem pedúnculo); peixe inteiro (pelo menos $70 \%$ do corpo); insetos, crustáceos; outros vertebrados; vegetais. A sobreposição alimentar foi calculada usando a sobreposição de Schoener (1970) adaptada por Agostinho et al. (2003), expressa em porcentagem, nos quais valores próximos de zero indicam pouca sobreposição alimentar e valores próximos de 100 significam dietas muito similares. Valores acima de $60 \%$ significam alta sobreposição. Este percentual de sobreposição é expresso pela seguinte equação:

$$
\mathrm{Pjk}=\left[\sum_{\mathrm{n}} \text { (mínimo pij, pik) }\right] * 100 \text { Onde: }
$$
espécie k;

Pjk = percentual de sobreposição entre espécie j e

pij, pik = proporção do recurso $\mathrm{i}$ em relação a todos os recursos usados pela espécie j e espécie $\mathrm{k}$ e

$\mathrm{n}=$ número de recursos alimentares.

Variações na atividade alimentar relacionadas ao ritmo circadiano, às classes de comprimento e aos ambientes foram verificadas empregando-se o grau de repleção médio (GR médio) (SANTOS, 1978). Para a análise da atividade alimentar em relação ao comprimento e visando facilitar comparações com outros trabalhos, os indivíduos foram agrupados nas seguintes classes de comprimento padrão (em cm): 2-4; >4-8; >8-16 e >16. Para a avaliação da atividade alimentar relacionada ao ritmo circadiano e aos ambientes, também foi utilizada a variação do Índice de Repleção Estomacal (IR), o qual representa a relação entre o peso do estômago e o peso total do indivíduo (SAnTos, 1978). Os dados de IR foram submetidos à análise de variância e teste de Tukey, utilizando o programa Statistica for Windows 5.0. Foi 
realizado o teste de Levene para testar a homogeneidade de variância. $O$ teste do $\chi^{2}$ (qui-quadrado) foi utilizado para verificar diferenças na captura entre pontos, ambientes e horários utilizando o programa BioEstat 3.0 (AYres et al., 2003).

\section{RESULTADOS}

Foram coletados 203 exemplares de $S$. maculatus sendo 164 em redes de espera, 22 em linhas e 17 nos arrastos. Destes, foram utilizados 199 para o estudo da alimentação sendo que 180 apresentaram algum conteúdo no estômago. Dos 86 exemplares de $P$. nattereri três foram coletados com uso de linha sendo os demais com uso de redes. Do total, 71 foram utilizados para o estudo da alimentação, sendo que 59 possuíam conteúdo no estômago. Doze exemplares foram depositados na coleção do Museu de Ciências e Tecnologia da PUCRS (MCP) (lotes MCP 26682; MCP 28912; MCP 28990; MCP 29192; MCP 31829; MCP 31830). Com relação ao número de indivíduos por ano, foram coletados apenas quatro exemplares de $P$. nattereri no primeiro ano de amostragem e 82 no segundo. Para S. maculatus foram capturados 82 exemplares no primeiro ano e 121 no segundo.

A análise da captura por ambiente sugere a preferência das espécies por ambientes lênticos, pois $77 \%$ $(\mathrm{n}=64)$ de $P$. nattereri $\left(\chi^{2}=24,39 ; \mathrm{p}<0,05 ; \mathrm{GL}=1\right)$ e $68 \%$ $(\mathrm{n}=112)$ de $S$. maculatus $\left(\chi^{2}=21,95 ; \mathrm{p}<0,05 ; \mathrm{GL}=1\right)$ foram capturadas nestes ambientes.

No que se refere à distribuição por ponto, houve uma diferença marcante entre a captura de $P$. nattereri no ponto 3 (próximo da foz) quando comparado aos outros dois $\left(\chi^{2}=148,50 ; p<0,05 ; G L=2\right)$. Cabe salientar que a coleta de indivíduos desta espécie nos pontos 1 e 2 ocorreu somente no segundo ano de amostragens: dezembro de 2000 e agosto de 2001 no ponto 2 (trecho intermediário) e em outubro de 2001 no ponto 1 (o mais distante da foz). Para $S$. maculatus o maior número de capturas também ocorreu no ponto $3\left(\chi^{2}=39,79 ; \mathrm{p}<0,05 ; \mathrm{GL}=2\right)$, embora tenha sido bem representada nos outros dois pontos (Fig. 1).

Quanto à variação do número de exemplares por horário, $P$. nattereri não apresentou variações significativas $\left(\chi^{2}=3,60 ; \mathrm{p}>0,05 ; \mathrm{GL}=1\right)$, enquanto $S$. maculatus apresentou o maior número de exemplares capturados na revisão da meia noite $\left(\chi^{2}=42,75 ; \mathrm{p}<0,05\right.$; GL=1) (Fig. 2).

A listagem dos organismos ingeridos pelas espécies (espectro trófico) encontra-se na Tabela I. Muitas espécies de peixes foram atacadas nas redes e tiveram suas partes arrancadas não tendo sido consideradas nesta análise. Provavelmente devido ao tamanho amostral e a presença de formas jovens, o espectro trófico de $S$. maculatus foi mais amplo que o de $P$. nattereri, o que sugere a necessidade de uma amostragem maior para a segunda espécie. Mesmo para a primeira, a análise de um número maior de estômagos de indivíduos das menores classes de tamanho seria necessária para resultados mais consistentes. Cinco espécies de peixes foram encontradas no estômago de cada espécie de piranha, sendo que Parapimelodus valenciennis (Lütken, 1874) e Acestrorhynchus pantaneiro (Menezes, 1992) foram consumidas por ambas. Na categoria restos de peixes, merece destaque a presença, por várias ocasiões, de barbilhões de Pimelodidae, provavelmente de $P$. valenciennis, um pequeno bagre planctófago que ocorre em águas abertas. Restos de peixes foi o item alimentar predominante em todas as classes, com exceção da classe 2-4 cm de $S$. maculatus, na qual os insetos e nadadeiras foram mais representativos (Tab. II)

Comparando-se o grau de repleção médio das duas espécies por horário verificou-se valores relativamente próximos (Fig. 3). A análise de variância não constatou diferenças significativas no índice de repleção (IR) entre os horários para $S$. maculatus $(\mathrm{F}=2,22 ; \mathrm{p}>0,05 ; \mathrm{GL}=3)$ e $P$. nattereri $(\mathrm{F}=0,26 ; \mathrm{p}>0,05 ; \mathrm{GL}=3)$. Com relação ao grau de repleção médio por classe de comprimento, não foram observadas diferenças significativas $(\mathrm{F}=0,28 ; \mathrm{p}>0,05$; $\mathrm{GL}=5)$, considerando as quatro classes de $S$. maculatus e duas de $P$. nattereri (Fig. 4). No que se refere ao IR entre as classes, a análise não foi significativa para $P$. nattereri $(\mathrm{F}=1,46 ; \mathrm{p}>0,05 ; \mathrm{GL}=1)$. Para $S$. maculatus, entretanto, constatou-se que a classe $4-8 \mathrm{~cm}$ apresentou $\mathrm{IR}=3,89$, significativamente maior que a classe $>16 \mathrm{~cm}$ com $\mathrm{IR}=2,08(\mathrm{~F}=4,22 ; \mathrm{p}<0,05 ; \mathrm{GL}=3)$. As médias de IR das classes $2-4 \mathrm{~cm}$ e $8-16 \mathrm{~cm}$ não foram estatisticamente diferentes das demais classes.

Uma análise do GR médio entre os ambientes demonstrou valores bastante próximos para as espécies. Na lagoa, S. maculatus apresentou GR médio de 1,75

Tabela I. Itens encontrados nos estômagos de Serrasalmus maculatus Kner, 1858 e Pygocentrus nattereri Kner, 1858 no rio Ibicuí, Rio Grande do Sul, entre dezembro de 1999 e janeiro de 2002 .

\begin{tabular}{|c|c|}
\hline Táxons & $\begin{array}{cc}S . & P . \\
\text { maculatus } & \text { nattereri }\end{array}$ \\
\hline
\end{tabular}

Crustáceos

OSTRACODA

$\mathrm{X}$

ISOPODA

CYMOTHOIDAE

Telotha henselii (von Martens, 1869) X

DECAPODA

TRICHODACTYLIDAE

Trichodactylus panoplus (von Martens, 1869) X PARASTACIDAE

Parastacus pilimanus (von Martens, 1869) X

PALAEMONIDAE

Macrobrachium sp.

Insetos

ODONATA

LIBELULLIDAE

AESCHNIDAE

EPHEMEROPTERA

POLYMITARCIDAE

DIPTERA

CHIRONOMIDAE

ORTHOPTERA

COLEOPTERA

Peixes

ENGRAULIDIDAE

Lycengraulis grossidens (Agassiz, 1829) X

CHARACIDAE

Astyanax jacuhiensis Cope, 1894

Bryconamericus stramineus Eigenmann, 1908 X

Aphyocharax anisitsi Eigenmann \& Kennedy, 1903

Odontostilbe pequira (Steindachner, 1882)

ACESTRORHYNCHIDAE

Acestrorhynchus pantaneiro Menezes, 1992 X $\quad$ X

LORICARIIDAE

PIMELODIDAE

Parapimelodus valenciennis (Lütken, 1874)

Aves

Mamíferos

RODENTIA

Estômagos com conteúdo
X

$\mathrm{X}$

$\mathrm{X}$

$\mathrm{X}$

$\mathrm{X}$

$\mathrm{X}$

$X$
$X$

X

X

X

$\mathrm{X}$

X

X X

X

$\mathrm{X}$

$\mathrm{X}$ 
$( \pm 1,05)$, enquanto que no rio, este foi de $2,03( \pm 1,03)$. Para $P$. nattereri o GR médio na lagoa foi de $1,79( \pm 1,21)$ e no rio de 2,28 ( $\pm 0,91)$. Quanto ao IR também não houve diferenças com relação aos ambientes para $S$. maculatus $(\mathrm{F}=1,50$; $\mathrm{p}>0,05 ; \mathrm{GL}=1)$ P. nattereri $(\mathrm{F}=2,95 ; \mathrm{p}>0,05 ; \mathrm{GL}=1)$.

A

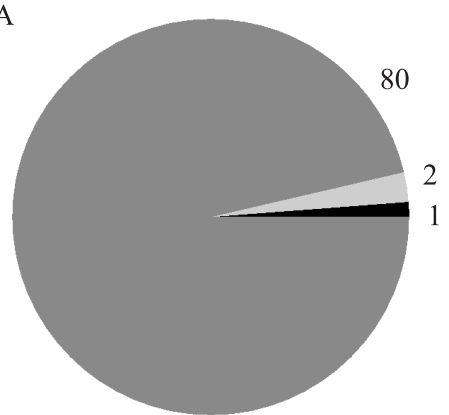

Quanto à sobreposição alimentar, foram constatados altos valores entre as espécies e entre as maiores classes de tamanho. As menores sobreposições ocorreram entre jovens de $S$. maculatus e adultos de $P$. nattereri (Tab. III).
B

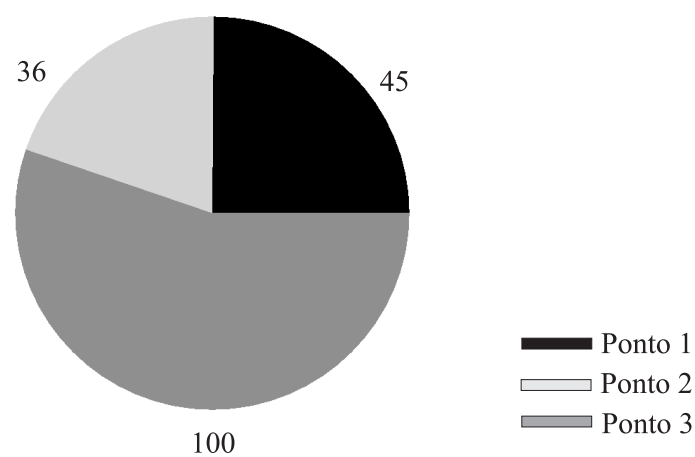

Fig. 1. Número de indivíduos de Pygocentrus nattereri Kner, 1858 (A) e Serrasalmus maculatus Kner, 1858 (B) coletados por ponto de amostragem no rio Ibicuí, Rio Grande do Sul, entre dezembro de 1999 e janeiro de 2002.

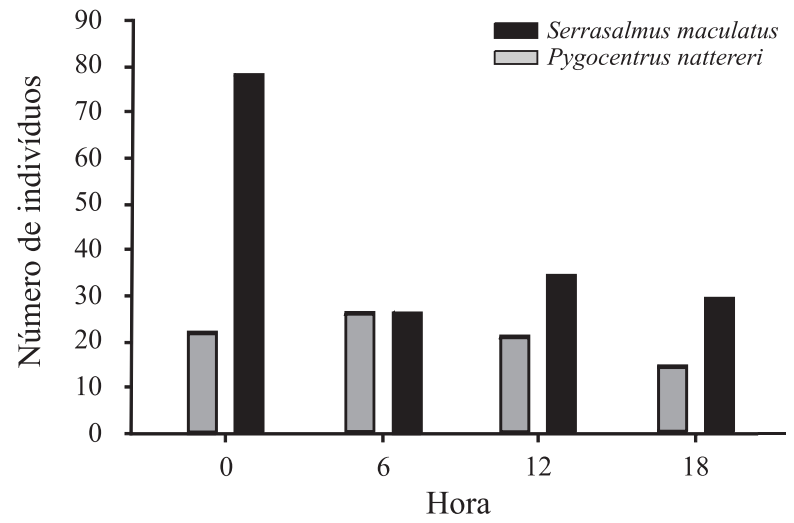

Fig. 2. Número de indivíduos de Pygocentrus nattereri Kner, 1858 e Serrasalmus maculatus Kner, 1858 capturados por horário nas redes de espera, no rio Ibicuí, Rio Grande do Sul, entre dezembro de 1999 e janeiro de 2002

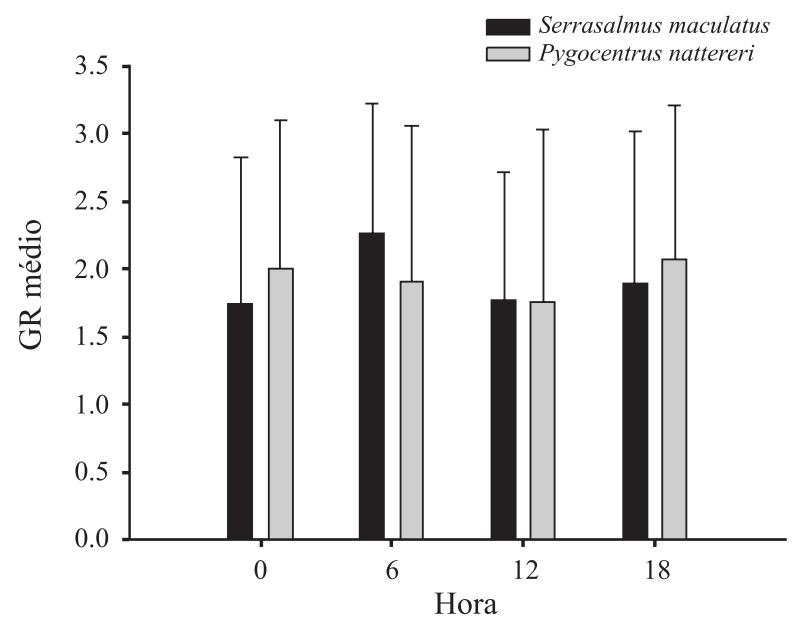

Fig. 3. Grau de repleção médio (GR médio) (+SD) de Pygocentrus nattereri Kner, 1858 e Serrasalmus maculatus Kner, 1858 nos diferentes horários de captura no rio Ibicuí, Rio Grande do Sul, entre dezembro de 1999 e janeiro de 2002.

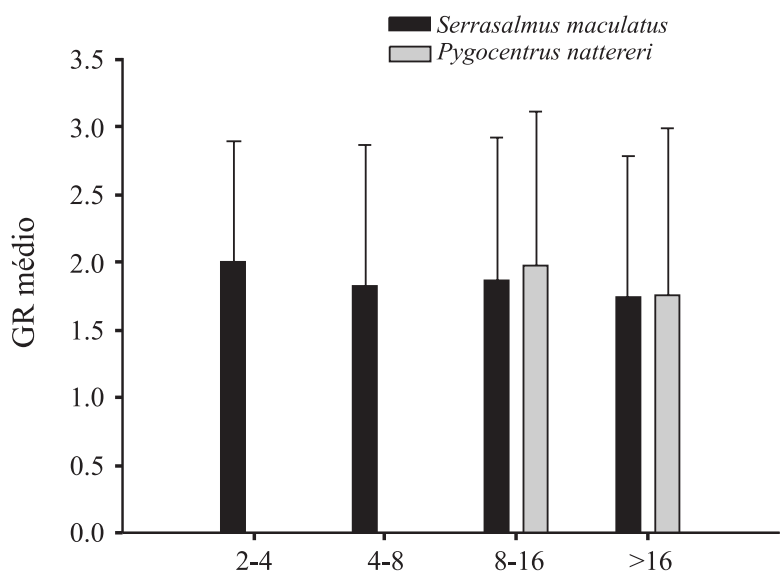

Classes de comprimento padrão $(\mathrm{cm})$

Fig. 4. Grau de repleção médio (GR médio) $(+\mathrm{SD})$ de Pygocentrus nattereri Kner, 1858 e Serrasalmus maculatus Kner, 1858 nas classes de comprimento padrão no rio Ibicuí, Rio Grande do Sul, entre dezembro de 1999 e janeiro de 2002.

Tabela II. Valores do Índice Alimentar (IAi) para as classes de comprimento $(\mathrm{cm})$ de Serrasalmus maculatus Kner, 1858 e Pygocentrus nattereri Kner, 1858 no rio Ibicuí, Rio Grande do Sul, entre dezembro de 1999 e janeiro de 2002.

\begin{tabular}{|c|c|c|c|c|c|c|c|}
\hline \multirow{2}{*}{$\begin{array}{l}\text { Categorias } \\
\text { alimentares }\end{array}$} & \multicolumn{4}{|c|}{ S. maculatus } & \multicolumn{3}{|c|}{ P. nattereri } \\
\hline & $2-4$ & $4-8$ & $8-16$ & $>16$ & $4-8$ & $8-16$ & $>16$ \\
\hline $\begin{array}{l}\text { Restos de } \\
\text { peixes }\end{array}$ & 3,49 & 85,98 & 98,87 & 97,45 & 92,08 & 97,84 & 99,16 \\
\hline Nadadeiras & 45,35 & 13,76 & 0,92 & 0,79 & & 0,45 & 0,01 \\
\hline Peixe inteiro & & & 0,05 & 0,63 & & 0,66 & \\
\hline Insetos & 50,58 & 0,12 & 0,06 & 0,77 & 7,92 & 0,01 & \\
\hline Crustáceos & 0,58 & & & 0,09 & & & \\
\hline $\begin{array}{l}\text { Outros } \\
\text { vertebrados }\end{array}$ & & & & & & 0,10 & 0,63 \\
\hline Vegetais & & 0,14 & 0,09 & 0,27 & & 0,95 & 0,20 \\
\hline $\begin{array}{l}\text { Estômagos com } \\
\text { conteúdo }(\mathrm{n})\end{array}$ & n 6 & 33 & 100 & 41 & 1 & 39 & 19 \\
\hline
\end{tabular}


Tabela III. Percentual de sobreposição alimentar de Schoener para as classes de comprimento (cm) de Serrasalmus maculatus Kner, 1858 (SER) e Pygocentrus nattereri Kner, 1858 (PYG) no rio Ibicuí, Rio Grande do Sul, entre dezembro de 1999 e janeiro de 2002 .

\begin{tabular}{lccccc}
\hline & PYG>16 & SER2-4 & SER4-8 & SER8-16 & SER>16 \\
\hline PYG8-16 & 77,15 & 41,06 & 72,53 & 78,44 & 71,36 \\
PYG>16 & & 25,79 & 57,26 & 78,56 & 55,73 \\
SER2-4 & & & 64,81 & 46,54 & 62,07 \\
SER4-8 & & & & 78,01 & 88,34 \\
SER8-16 & & & & & 70,60 \\
\hline
\end{tabular}

\section{DISCUSSÃO}

Quanto ao número de indivíduos, S. maculatus foi mais abundante que $P$. nattereri. Esta última espécie só foi registrada recentemente para o alto rio Uruguai e em número bem menor que S. maculatus (E. Zaniboni-Filho, com. pess.). BERTOLETTI et al. (1989; 1990) haviam registrado somente $S$. maculatus no alto Uruguai e na área da futura usina hidrelétrica de Garabi. Serrasalmus maculatus também foi a única espécie de piranha capturada em amostragens realizadas em um trecho do alto rio Uruguai, entre maio de 1997 e maio de 1999 (Lisiane Hahn, com. pess.). Trabalhos realizados nos formadores do rio Ibicuí (rios Ibicuí-Mirim e Santa Maria) não constataram a presença de P. nattereri (WeIs et al., 1983; BOSSEMEYER et al., 1985).

A preferência das piranhas por ambientes lênticos já é bem conhecida (BERTOLETTI, 1985; AgostinHO \& JúLIO JR., 2002). O rio Ibicuí tem por característica ser um rio de pouca correnteza, com muitas áreas de remanso. A captura de indivíduos em ambiente lótico, bem menor do que nas lagoas, pode ter sido influenciada pelo fato de que em algumas ocasiões as coletas foram realizadas em águas mais calmas.

Sazima \& Machado (1990) relataram que esta espécie foi preferencialmente diurna em estudos realizados na região do Pantanal, mas indivíduos maiores que $120 \mathrm{~mm}$ estendiam sua atividade até o início da noite (aproximadamente até às 21h). AgostinHo et al. (2003) também obtiveram altas taxas de captura de $S$. maculatus no período entre 16 e $20 \mathrm{~h}$. No presente estudo, houve uma maior captura de $S$. maculatus na revisão da meianoite. Como no verão a revisão das $18 \mathrm{~h}$ foi realizada ainda com luminosidade, nesta estação os peixes retirados das redes na revisão da meia-noite incluem aqueles em atividade ao anoitecer. Embora sem diferenças marcantes, a captura de $P$. nattereri concorda com as observações de Sazima \& Machado (1990), onde indivíduos de tamanho médio a grande forrageiam mais ao amanhecer, entardecer e à noite (até às $22 \mathrm{~h}$ ).

$\mathrm{O}$ espectro trófico de $S$. maculatus neste estudo mostrou-se bem mais amplo que o de P. nattereri. O maior número de exemplares analisados da primeira espécie pode ter contribuído para este resultado. PIORSKI et al. (2005) relatam um espectro mais variado para Serrasalmus aff. brandtii (Lütken, 1875) quando comparada à $P$. nattereri no lago de Viana, no Maranhão. Naquele estudo, apenas peixes e vegetais estiveram presentes (ao longo de todos os meses de amostragem) na dieta de $P$. nattereri, enquanto que para $S$. aff. brandtii efemerópteros também foram encontrados em todos os meses (além de peixes e vegetais), o que sugere uma maior especialização de $P$. nattereri.

O grau médio de repleção e o índice de repleção não apresentaram variações significativas entre ambientes, horários ou classes de comprimento para $P$. nattereri. Para S. maculatus foram constatadas diferenças significativas de IR entre as classes $4-8 \mathrm{~cm}$ e $>16 \mathrm{~cm}$, evidenciando uma maior repleção estomacal nos indivíduos menores. $O$ fato da classe $2-4 \mathrm{~cm}$ não ter sido significativamente diferente das maiores pode estar relacionada com o baixo número de estômagos analisados $(n=6)$, ou pelo fato da maior contribuição ser de insetos, que apresentam uma digestibilidade mais lenta devido ao exoesqueleto.

A categoria alimentar que apresentou os maiores valores do Índice Alimentar foi "restos de peixes". Este padrão também foi encontrado por AgostinHo et al. (2003). Provavelmente uma parte do item "restos de peixes" foi ingerida a partir de indivíduos que estavam presos nas redes de espera. $\mathrm{O}$ ataque a peixes nas redes também é citado por Agostinho et al. (1997) e certamente ajuda a aumentar o GR das amostras onde ocorre.

A ingestão de peixes inteiros ocorreu com mais freqüência nas maiores classes de comprimento (exceção para $P$. nattereri $>16 \mathrm{~cm}$ ) e foi constituída principalmente por pequenos Characiformes. Pompeu (1999) identificou onze espécies de peixes nos estômagos de Serrasalmus brandtii em quatro lagoas marginais do rio São Francisco, sendo uma boa parte constituída de caracídeos de pequeno porte ingeridos inteiros. BISTONI \& HARO (1995) conseguiram identificar quatro espécies de peixes no conteúdo de S. maculatus no rio Dulce, Argentina.

Muitos autores utilizam a categoria "escamas" separada das demais (MACHADO-AlliSON \& GARCIA, 1986; Nico \& TAPHORN, 1988; SAZIMA \& MACHADO, 1990). Neste trabalho entretanto, as escamas foram incluídas na categoria "restos de peixes", conforme AgostinHo et al. (2003). Embora tenham sido encontradas em muitos dos conteúdos, é difícil saber se foram ingeridas junto com pedaços de musculatura das presas ou sozinhas. Porém, em alguns estômagos foram encontradas apenas escamas, algumas grandes para o tamanho do predador. Isto sugere que as espécies apresentam atividade lepidofágica, mas esta não parece ser muito intensa. BISTONI \& HARO (1995) consideraram a ingestão de escamas como ocasional para S. maculatus nos banhados do rio Dulce, Argentina. SAZIMA \& MACHADO (1990) registraram uma frequiência de ocorrência de $29,1 \%$ para este item em $P$. nattereri, no Pantanal Mato-Grossense.

A ingestão de nadadeiras por S. maculatus é citada por vários autores (NoRTHCOTE et al., 1986, 1987; SAZIMA \& Pombal-JR., 1988; SAZIMA \& MACHADO, 1990; BistonI \& Haro, 1995; Agostinho et al., 2003). Os dados de IAi obtidos para $S$. maculatus demonstram que sua importância na dieta foi diminuindo com o aumento de tamanho dos peixes, fato também verificado por Agostinho et al. (2003). Em P. nattereri as nadadeiras tiveram um IAi ainda menor. SAZIMA \& MACHADO (1990) não encontraram nadadeiras no conteúdo estomacal de 24 espécimes com comprimento padrão variando de 8 a $24 \mathrm{~cm}$, no Pantanal Mato-Grossense. 
Os insetos foram o principal alimento de $S$. maculatus da classe 2-4 cm. Seu consumo ocorre também nas classes maiores, porém com menor importância. Dados obtidos por outros autores indicam que a utilização deste recurso varia bastante em função do local e da época do ano. Sazima \& Machado (1990) encontraram insetos em 69,2\% dos estômagos de S. maculatus com 6,4-16,0 cm de comprimento na região do Pantanal. No mesmo estudo, os autores registraram um percentual menor $(12,5 \%)$ para $P$. nattereri de $8-24 \mathrm{~cm}$. Por outro lado, Agostinho et al. (2003) encontraram um valor de IAi para insetos de apenas 0,02 para $S$. maculatus de 2-4 $\mathrm{cm}$, na região do alto rio Paraná.

No presente estudo, a ingestão de partes de vertebrados (aves e mamíferos) foi registrada somente para $P$. nattereri. BonetTo et al. (1967) e BRAGA (1981) afirmam que $P$. nattereri se alimenta de peixes inteiros ou em pedaços, mas também utiliza insetos, crustáceos, moluscos, pequenos vertebrados e plantas. Indivíduos adultos de Pygocentrus cariba (Humboldt \& Valenciennes, 1821) ingeriram pedaços de outros vertebrados (quelônios, aves e mamíferos) no período de águas altas nos Llanos da Venezuela, quando os peixes estavam muito dispersos (WINEMILLER, 1989).

A ocorrência de itens vegetais no trato digestivo de piranhas é tida como acidental para algumas espécies (NICO \& TAPHORN, 1988) enquanto para outras, faz parte da dieta (Goulding, 1980; Machado-Allison \& Garcia, 1986). No rio Ibicuí, tanto $S$. maculatus como $P$. nattereri apresentaram vegetais em seus conteúdos estomacais, mas os baixos valores de IAi sugerem uma ingestão acidental. Baixos valores de IAi para vegetais também foram registrados por Agostinho et al. (2003) para $S$. maculatus.

Apesar do pequeno número de indivíduos das menores classes de comprimento, foi possível constatar a ingestão de microcrustáceos por jovens de $S$. maculatus. A utilização de microcrustáceos por esta espécie, em sua fase inicial de desenvolvimento, já havia sido constatada por SAZIMA \& ZAMPROGNO (1985). Crustáceos maiores, como camarões e caranguejos, também foram registrados para indivíduos grandes desta espécie, porém com baixo IAi (SAZIMA \& MACHADO, 1990). Para Serrasalmus rhombeus (Linnaeus, 1766), no açude Lima Campos (Ceará), camarão foi o item mais importante (BRAGA, 1954)

O padrão de alimentação diário não apresentou diferenças significativas entre os horários para ambas as espécies. AgostinHo et al. (2003) constataram que adultos de $S$. maculatus se alimentam continuamente, enquanto os jovens apresentaram um pico alimentar entre às $12 \mathrm{e}$ 16h. A análise separada de jovens e adultos não foi realizada pelo pequeno número de jovens em alguns horários.

O elevado grau de sobreposição alimentar verificado para $S$. maculatus e $P$. nattereri no rio Ibicuí deve ter sido influenciado pela predação realizada aos peixes presos nas redes de pesca, fazendo com que a categoria "restos de peixes" apresentasse alto IAi. A sobreposição alimentar, dentro da mesma espécie, entre classes de comprimento próximas, também foi elevada. Altas taxas de sobreposição alimentar foram registradas para $S$. maculatus e $S$. marginatus no alto rio Paraná (Agostinho et al., 2003) e para duas espécies de Serrasalmus que se alimentam preferencialmente de nadadeiras de peixes nos Llanos da Venezuela (Winemiller, 1989). Pygocentrus nattereri foi capturada no mesmo ambiente que $S$. maculatus em 14 das 15 amostragens. Isto demonstra que as duas espécies ocupam os mesmos habitats ao mesmo tempo, aumentando a possibilidade de competição.

Por outro lado, a utilização de restos de peixes pode aumentar indevidamente o índice de sobreposição alimentar, visto que não há como saber quais as espécies foram consumidas pelas piranhas. Analisando o espectro alimentar, somente duas espécies de peixes foram consumidas por ambas. Levando isto em conta, a sobreposição alimentar entre as duas espécies pode ser baixa. Todavia, a maior abundância de $P$. nattereri na parte inferior do rio Ibicuí, próximo ao rio Uruguai, pode ser indicativo da competição com S. maculatus nos trechos superiores do rio.

O pequeno número (ou ausência) de exemplares das menores classes de tamanho (entre 2-4 e 4-8 $\mathrm{cm}$ de comprimento padrão) impede uma análise da alimentação destas espécies em sua fase inicial de desenvolvimento. Deste modo, a utilização de arrastos em lagoas com maior quantidade de vegetação aquática deveria ser utilizada para a captura de indivíduos destas classes. Para as demais classes de comprimento, os resultados obtidos no rio Ibicuí seguem os padrões encontrados para outras bacias hidrográficas, denotando seu hábito piscívoro generalista.

Agradecimentos. Os autores agradecem a todas as pessoas que colaboraram durante a coleta de material e nos trabalhos de laboratório. ERB agradece especialmente à Roberto E. Reis pela orientação durante o curso de Doutorado na PUCRS e sugestões ao manuscrito; ao CNPq pela concessão da bolsa e a Bernardo Baldisserotto, Clarice B. Fialho, Emiko K. de Resende, Nelson F. Fontoura, Carlos S. Agostinho, Marilise M. Krügel e a um revisor anônimo pelas sugestões.

\section{REFERÊNCIAS BIBLIOGRÁFICAS}

Agostinho, C. S.; Agostinho, A. A.; Marques, E. E. \& Bini, L. M. 1997. Abiotic factors influencing piranha attacks on netted fish in the upper Paraná River, Brazil. North American Journal of Fisheries Management 17:712-718.

Agostinho, C. S.; Hahn, N. S. \& Marques, E. E. 2003. Patterns of food resource use by two congeneric species of piranhas (Serrasalmus) on the upper Paraná River floodplain. Brazilian Journal of Biology 63(2):177-182.

Agostinho, C. S. \& Júlio JR., H. F. 2002. Observation of an invasion of the piranha Serrasalmus marginatus Valenciennes, 1847 (Osteichthyes, Serrasalmidae) into the Upper Paraná River. Acta Scientiarum 24(2):391-395.

Agostinho, C. S. \& Marques, E. E. 2001. Selection of netted prey by piranhas, Serrasalmus spilopleura and $S$. marginatus (Pisces, Serrasalmidae). Acta Scientiarum 23(2):461-464.

Almeida, V. L. L. DE; Hahn, N. S. \& Agostinho, C. S. 1998. Stomach content of juvenile and adult piranhas (Serrasalmus marginatus) in the Paraná floodplains, Brazil. Studies on Neotropical Fauna \& Environment 33:100-105.

Alvim, M. C. C.; Oliveira, A. K.; Alves, C. B. M. \& Peret, A. C 1999. Diet of young Serrasalmus brandtii (Teleostei: Serrasalminae) from the Cajuru hydroeletric plant reservoir (MG, Brazil), in relation to the vegetal biomass in the depletion zone. Multiciência 3(2):94-103.

Ayres, M.; Ayres Jr., M.; Ayres, D. M. \& Santos, A. S. dos. 2003. BioEstat 3.0: aplicações estatísticas nas áreas das 
ciências biológicas e médicas. Belém, Sociedade Civil Mamirauá. 290p.

Beheregaray, R. C. P.; Querol, E.; Gonçalves, J.; Soto, J. M. R. \& CARvalho, A. D. 2000. Fauna microbiana ocorrente na cavidade bucal da piranha Serrasalmus spilopleura (Characidae) no município de Uruguaiana, Rio Grande do Sul, Brasil. Revista da Faculdade de Zootecnia Veterinária e Agronomia de Uruguaiana 7/8(1):59-61.

Beheregaray, R. C. R.; Querol, E.; Carvalho, A. D.; Silveira, F. S. \& Мота, I. C. 2001. Piranha Serrasalmus spilopleura (Characidae) sobrevivendo sem nadadeiras peitorais. Biotemas 14(1):141-145.

Bertoletti, J. J. 1985. Aspectos sistemáticos e biológicos da ictiofauna do rio Uruguai. Veritas 30(117):93-127.

Bertoletti, J. J.; Lucena, C. A. S.; Lucena, Z. M. S.; Malabarba, L. R. \& ReIs, R. E. 1989. Ictiofauna do rio Uruguai superior entre os municípios de Aratiba e Esmeralda, Rio Grande do Sul, Brasil. Comunicações do Museu de Ciências da PUCRS (48):3-42.

Bertoletti, J. J.; Lucena, C. A. S.; Lucena, Z. M. S.; Malabarba, L. R. \& ReIs, R. E. 1990. Estrutura e composição da fauna de peixes na área de influência da futura Usina Hidrelétrica de Garabi, Rio Grande do Sul, Brasil. Comunicações do Museu de Ciências da PUCRS 3(2):33-97.

Bistoni, M. De L. A. \& Haro, J. G. 1995. Hábitos alimentarios de Serrasalmus spilopleura (Pisces, Serrasalmidae) en los bañados del rio Dulce (Córdoba, Argentina). Revista Brasileira de Biologia 55(4):847-853.

Bonetto, A.; Pignalberi, C. \& Cordiviola, E. 1967. Las "palometas" o "piranhas" de las águas del Paraná medio. Acta Zoologica Lilloana 23:45-65.

Bossemeyer, I. M. K.; Weis, M. L. C.; Bennemann, S. T. \& Bier, M. L. S. 1985. Ictiofauna do Rio Santa Maria, RS. Ciência e Natura 7:209-222.

Braga, R. A. 1954. Alimentação de pirambeba, "Serrasalmus rhombeus" (L. 1766) Lacépède; 1803, no açude Lima Campos, Icó, Ceará. Revista Brasileira de Biologia 14(4):477-492. 1981. Ecologia e etologia das piranhas do nordeste do Brasil (Pisces - Serrasalmus Lacépède, 1803). Fortaleza, DNOCS. 268p.

Fagundes, C. K.; Behr, E. R. \& Kotzian, C. B. 2008. Diet of Iheringichthys labrosus (Siluriformes, Pimelodidae) in the Ibicuí river, southern Brazil. Iheringia, Série Zoologia, 98(1):60-65.

Goulding, M. 1980. The fishes and the forest. Explorations in Amazonian natural history. Berkeley, University of California. 280p.

Hyslop, E. J. 1980. Stomach contents analysis: a review of methods and their application. Journal Fish Biology 17(4):411-429.

JÉGu, M. 2003. Subfamily Serrasalminae. In: Reis, R. E.; Kullander, S. O. \& Ferraris JR., C. J. orgs. Check List of Freshwater Fishes of South and Central America. Porto Alegre, EDIPUCRS. p.182-196.

Kawakami, E. \& Vazzoler, G. 1980. Método gráfico e estimativa de índice alimentar aplicado ao estudo de alimentação de peixes. Boletim do Instituto Oceanográfico 29:205-207.

LeÃo, E. L. M.; Leite, R. G.; Chaves, P. T. C. \& Ferraz, E. 1991. Aspectos da reprodução, alimentação e parasitofauna de uma espécie rara de piranha Serrasalmus altuvei, Ramíres, 1965 (Pisces: Serrasalmidae) do baixo rio Negro. Revista Brasileira de Biologia 51(3):545-553.

Machado-Allison, A. \& Garcia, C. 1986. Food habits and morphological changes during ontogeny in three Serrasalmin species of Venezuelan flood plain. Copeia 1986(1):93-96.

Magalhães, E. M.; Almeida, R. G.; Gurgel, H. C. B. \& Barbieri, G. 1990. Contribuição ao estudo da alimentação de Serrasalmus brandtii (Reinhardt, 1874) (Characiformes, Serrasalmidae) do rio Piranhas-Açu, Pendências, Rio Grande do Norte. Revista Ceres 37:429-442.

Malabarba, L. R. \& Reis, R. E. 1987. Manual de técnicas para a preparação de coleções zoológicas. 36. Peixes. Campinas, Sociedade Brasileira de Zoologia. 14p.

Myers, J. S. 1972. The piranha book. Neptune City, TFH. $128 \mathrm{p}$.

Nico, L. G. 1990. Feeding chronology of juvenile piranhas, Pygocentrus notatus, in the Venezuelan llanos. Environmental Biology of Fishes 29:51-57.

Nico, L. G. \& TAPHORN, D. C. 1988. Food habits of piranhas in the low 1lanos of Venezuela. Biotropica 20:311-321.

Northcote, T. G.; Arcifa, M. S. \& Froehlich, O. 1987. Fin-feeding by the piranha (Serrasalmus spilopleura Kner): the cropping of a novel renewable resource. In: Congress Europ. Ichthyology, 5. 1985, Stockholm. Proceedings of 5th Congress Europ. Ichthyology. Stockholm, Swedish Museum of Natural History. p.133-143.

Northcote, T. G.; Northcote, R. G. \& Arcifa, M. S. 1986 Differential cropping of the caudal fin lobes of prey fishes by the piranha, Serrasalmus spilopleura Kner. Hydrobiologia 141:199-205.

Oliveira, A. K.; Alvim, M. C. C.; Peret, A. C. \& Alves, C. B. M. 2004. Diet shifts related to body size of the pirambeba Serrasalmus brandtii Lütken, 1875 (Osteichthyes, Serrasalminae) in the Cajuru reservoir, São Francisco River basin, Brazil. Brazilian Journal of Biology 64(1):117-124.

Piorski, N. M.; Alves, J. R. L.; Machado, M. R. B. \& Correia, M. M. F. 2005. Alimentação e ecomorfologia de duas espécies de piranhas (Characiformes: Characidae) do lago de Viana, estado do Maranhão, Brasil. Acta Amazônica 35(1):63-70.

Pompeu, P. S. 1999. Dieta da pirambeba Serrasalmus brandtii Reinhardt (Teleostei, Characidae) em quatro lagoas marginais do rio São Francisco, Brasil. Revista Brasileira de Zoologia 16(supl. 2):19-26.

SANTos, E. P. DOS. 1978. Dinâmica de populações aplicada à pesca e piscicultura. São Paulo, Hucitec. 129p.

SAZIMA, I. \& MAChADO, F. A. 1990. Underwater observations of piranhas in western Brazil. Environmental Biology of Fishes 28: $17-31$.

Sazima, I. \& Pombal JR., J. P. 1988. Mutilação de nadadeiras em acarás, Geophagus brasiliensis, por piranhas, Serrasalmus spilopleura. Revista Brasileira de Biologia 48:477-483.

SAZima, I. \& ZAMProgno, C. 1985 . Use of water hyacinths as shelter, foraging place, and transport by young piranhas, Serrasalmus spilopleura. Environmental Biology of Fishes 12(3):237-240.

Schoener, T. W. 1970. Non-synchronous spatial overlap of lizards in patchy habitats. Ecology 51:408-418.

Sverlij, S. B.; Shenke, R. L. D.; Lopez, H. L. \& Espinach-Ros, A. 1998. Peces del rio Uruguay. Montevideo, CARU. 89p.

Weis, M. L. C.; Bossemeyer, I. M. K.; Bier, M. DE L. \& LipPold, H. 1983. Inventário da fauna ictiológica do Rio Ibicuí-Mirim, RS. Ciência \& Natura 5:135-152.

Winemiller, K. O. 1989. Ontogenetic diet shifts and resouce partitioning among piscivorous fishes in the Venezuelan llanos. Environmental Biology of Fishes 26:177-199.

Zavala-Camin, L. A. 1996. Introdução aos estudos sobre alimentação natural em peixes. Maringá, EDUEM. 129p.

Recebido em outubro de 2007. Aceito em julho de 2008. ISSN 0073-4721

Artigo disponível em: www.scielo.br/isz 